\title{
Early applications of the $R$-matrix SAMMY code for charged-particle induced reactions and related covariances
}

\author{
Marco T. Pigni ${ }^{\mathrm{a}, *}$, Ian C. Gauld, and Stephen Croft \\ Oak Ridge National Laboratory, Oak Ridge, TN, USA
}

\begin{abstract}
The SAMMY code system is mainly used in nuclear data evaluations for incident neutrons in the resolved resonance region (RRR), however, built-in capabilities also allow the code to describe the resonance structure produced by other incident particles, including charged particles. $(\alpha, \mathrm{n})$ data provide fundamental information that underpins nuclear modeling and simulation software, such as ORIGEN and SOURCES4C, used for the analysis of neutron emission and definition of source emission processes. The goal of this work is to carry out evaluations of charged-particle-induced reaction cross sections in the RRR. The SAMMY code was recently used in this regard to generate a Reich-Moore parameterization of the available ${ }^{17,18} \mathrm{O}(\alpha, \mathrm{n})$ experimental cross sections in order to estimate the uncertainty in the neutron generation rates for uranium oxide fuel types. This paper provides a brief description of the SAMMY evaluation procedure for the treatment of ${ }^{17,18} \mathrm{O}(\alpha, \mathrm{n})$ reaction cross sections. The results are used to generate neutron source rates for a plutonium oxide matrix.
\end{abstract}

\section{Introduction}

Evaluated nuclear data describing charged-particle interactions are essential for a large range of applications. Among these, $\alpha$-particle induced reactions on light nuclei are of particular importance to calculate neutron emission via $(\alpha, n)$ process as in neutron-based verification measurements of unirradiated uranium and plutonium nuclear materials in a non-metal matrix. Moreover, in irradiated nuclear fuels that achieve a moderate to high burnup, the neutron source is typically dominated by ${ }^{242,244} \mathrm{Cm}$ spontaneous fission. However, in low-burnup fuels such as those encountered in weapons material production, the $(\alpha, n)$ neutron processes can represent a large component of the total neutron source and are therefore important to neutron measurement techniques. Moreover, the recent revival of research on nuclear data covariances has lead to use these type of nuclear data in applications. The ultimate need is to estimate the accuracy of applied quantities, e.g. neutron source rates, due to the uncertainties in basic data.

Currently, the ENDF/B-VII.1 nuclear data library [1] contains no information on $\alpha$-particle induced cross

\footnotetext{
a e-mail: pignimt@ornl.gov

* Notice: This manuscript has been authored by UT-Battelle, LLC under Contract No. DE-AC05-00OR22725 with the U.S. Department of Energy. The United States Government retains and the publisher, by accepting the article for publication, acknowledges that the United States Government retains a nonexclusive, paid-up, irrevocable, world-wide license to publish or reproduce the published form of this manuscript, or allow others to do so, for United States Government purposes. The Department of Energy will provide public access to these results of federally sponsored research in accordance with the DOE Public Access Plan (http://energy.gov/ downloads/doe-public-access-plan).
}

section and covariance data. The ultimate goal of this work is to carry out evaluations of $\alpha$-particle induced cross sections, including their related covariance information for a comprehensive set of nuclei. This would provide fundamental information that underpins nuclear modeling and simulation software, such as ORIGEN [2] and SOURCES4C [3,4], which are widely used for the analysis of neutron emission and source emission processes, but they are also used in nuclear safeguards and spent nuclear fuel characterization. In this regard, the SAMMY code [5] was recently used to generate a Reich-Moore parameterization of the ${ }^{17,18} \mathrm{O}(\alpha, \mathrm{n})$ available experimental cross sections in order to estimate the uncertainty in the neutron generation rates for uranium oxide fuel types [6].

The most recent version of the $R$-matrix code SAMMY allows the study of the ingoing and outgoing chargedparticle channels in the low-energy interaction range. Although the SAMMY code system is mainly used in nuclear data evaluations for incident neutrons in the RRR, built-in capabilities also allow the code to describe the resonance structure produced by other incident particles, including charged particles. Focusing on ${ }^{17,18} \mathrm{O}(\alpha, \mathrm{n})$ cross sections in the RRR, this work describes the preliminary results of the SAMMY methodology for the treatment of $\alpha$-induced cross sections.

As in Babut's analysis [7] that, although, did not focus on estimating cross section covariances, the evaluation methodology uses the Reich-Moore approximation to compute the ${ }^{17,18} \mathrm{O}(\alpha, \mathrm{n})$ reaction cross sections and the Bayesian fitting method to generate a set of resonance parameters with a related covariance matrix in the incident $\alpha$ energy range up to $5 \mathrm{MeV}$. These two oxygen isotopes are of essential importance for neutron source analysis because they are responsible for the total neutron emission via $(\alpha, n)$ in the relevant energy range between $0-7 \mathrm{MeV}$. In particular, the ${ }^{18} \mathrm{O}$ isotope, having an isotopic abundance 
$(0.205 \%)$ one order of magnitude larger than that for ${ }^{17} \mathrm{O}$ $(0.038 \%)$, dominates neutron emission via $(\alpha, \mathrm{n})$ reactions. In the energy range below $7 \mathrm{MeV}$, the most abundance isotope ${ }^{16} \mathrm{O}(99.757 \%)$ does not contribute to neutron emission process simply because its threshold energy for the $(\alpha, \mathrm{n})$ reaction is $E_{\mathrm{th}}=15.1 \mathrm{MeV}$.

The objective of this paper is to present a brief description of the SAMMY method applied to compute $(\alpha, \mathrm{n})$ cross sections in the RRR applied to isotopes essentially important to application as ${ }^{17,18} \mathrm{O}$. As an example we show the distribution of the neutron source rate for a plutonium oxide matrix generated by the $(\alpha, n)$ reactions and its source uncertainty obtained from the related covariance information.

The paper is organized as follows. The evaluation methodology is outlined in Sect. 2, the calculations and results are presented and discussed in Sect. 3 followed by the application of the results in Sect. 4. Our conclusions are detailed in Sect. 5.

\section{Methodology}

This methodology is based on the SAMMY code system, a legacy tool for calculating (neutron but also chargedparticle induced) cross sections used for nuclear data evaluations in the RRR. The code incorporates selected $R$-matrix approximations coupled to the Bayesian method in order to fit experimental data and ultimately to generate a set of resonance parameters with related covariance matrix. For this task the results were based on the ReichMoore approximation, which defines the expression for elastic and reaction cross sections better than other singleand multi-level variants of the $R$-matrix theory.

For generating the various reaction cross sections $\sigma_{c c^{\prime}}$ for an incoming channel $c$ and outgoing channel $c^{\prime}$, the SAMMY algorithm is based on the computation of the scattering matrix whose elements can be written as [5]

$$
X_{c c^{\prime}}=\sqrt{P_{c}} L_{c}^{-1} \sum_{c^{\prime \prime}}\left[\left(L^{-1}-R\right)^{-1}\right]_{c c^{\prime \prime}} R_{c^{\prime \prime} c^{\prime}} \sqrt{P_{c^{\prime}}} \delta_{J J^{\prime}}
$$

where, in the eliminated-channel approximation, the matrix $R$ (or $R$-matrix) for the channel spin group defined by the total spin $J^{\pi}$ (with parity $\left.\pi=\left(-1^{\ell}\right) \pi_{i} \pi_{I}\right)$ is

$$
R_{c c^{\prime}}=\left[\sum_{\lambda=1}^{n} \frac{\gamma_{\lambda c} \gamma_{\lambda c^{\prime}}}{E_{\lambda}-E-{ }_{l} \bar{\Gamma}_{\lambda \gamma} / 2}+R_{c}^{\mathrm{ext}} \delta_{c c^{\prime}}\right] \delta_{J J^{\prime}} .
$$

From the best fit of experimental data SAMMY computes the set of $n$ reduced-width amplitudes $\gamma_{\lambda c}$ related to the channel width using

$$
\Gamma_{\lambda c}=2 \gamma_{\lambda c}^{2} P_{c}(E)
$$

where the penetrability factors depend on the regular and irregular Coulomb functions $F_{\ell}$ and $G_{\ell}$, respectively, as $P_{c}(E)=k_{c} a_{c} /\left[F_{\ell}^{2}\left(k_{c} a_{c} ; \eta\right)+G_{\ell}^{2}\left(k_{c} a_{c} ; \eta\right)\right]$ calculated at the corresponding channel wave-number $k_{c} \equiv k_{c}(E)$ and channel radius $a_{c}$ for the $\ell$-th partial wave. In this work the quantity $L_{c}=\left(S_{c}-B_{c}\right)+$ " $P_{c}$ was purely imaginary based on the choice of an energy-dependent boundary condition $B_{c}=S_{c}$ being $S_{c}$ the shift factor.

In the channel notation, the index $c$ can be related to a specific partition: elastic scattering ${ }^{17} \mathrm{O}(\alpha, \alpha){ }^{17} \mathrm{O}$

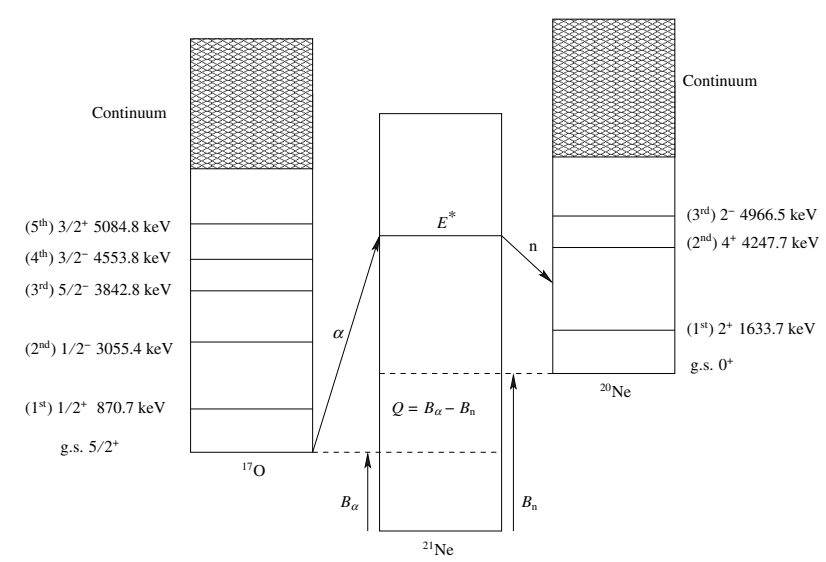

Figure 1. Reaction scheme for $\mathrm{n}+{ }^{17} \mathrm{O}$ cross sections including neutron emission. $B_{\alpha}$ and $B_{n}$ are, respectively, the $\alpha$-particle and neutron binding energies.

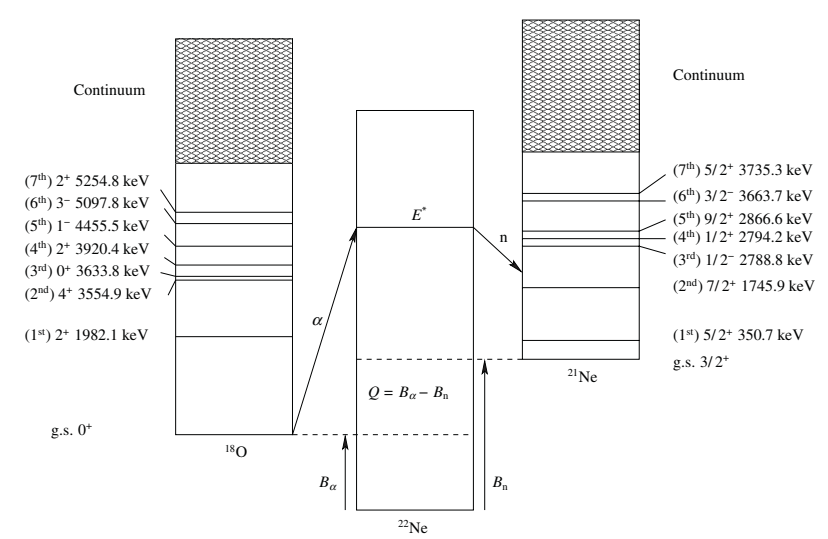

Figure 2. Reaction scheme for $\mathrm{n}+{ }^{18} \mathrm{O}$ cross sections including neutron emission. $B_{\alpha}$ and $B_{n}$ are, respectively, the $\alpha$-particle and neutron binding energies.

or reaction ${ }^{17} \mathrm{O}(\alpha, \mathrm{n}){ }^{21} \mathrm{Ne}$ with residual nucleus in the ground state (g.s.) are fully specified by the total angular momentum $J$ and the channel spin $s$ obtained by the quantum-mechanical triangle relations $|\ell-s| \leq J \leq \ell+$ $s$ and $|I-i| \leq s \leq I+i$, respectively. As shown in Figs. 1 and 2, depending on the incident energy of the particle with spin $i$, each partition can be also specified for the target and/or residual nucleus (with spin $I$ ) in the ground state or in one of its excited states.

Up to the $2^{\text {nd }}$ excited state of ${ }^{20} \mathrm{Ne}\left(E_{\mathrm{th}}=4.19 \mathrm{MeV}\right)$ was included in this work for the $n+{ }^{17} \mathrm{O}$ interaction system, and up to the $5^{\text {th }}$ excited state of ${ }^{21} \mathrm{Ne}\left(E_{\mathrm{th}}=4.35 \mathrm{MeV}\right)$ was included for the $\mathrm{n}+{ }^{18} \mathrm{O}$ interaction system. For both target nuclei ${ }^{17} \mathrm{O}$ and ${ }^{18} \mathrm{O}$ we limited our analysis to elastic scattering. The assumption of excluding the alpha inelastic scattering was based on the calculation of the chargedparticle penetrability factors. Although inelastic scattering thresholds based on mass consideration occur at $870.7 \mathrm{keV}$ for ${ }^{17} \mathrm{O}$ (Fig. 1) and $1.982 \mathrm{MeV}$ for ${ }^{18} \mathrm{O}$ (Fig. 2), their effective thresholds are at incident $\alpha$ energies approaching the Coulomb barrier $\left(\approx 4.7 \mathrm{MeV}\right.$ for $\left.{ }^{18} \mathrm{O}\right)$ for which the magnitude of the penetrability factors is noticeable.

\section{Results}

A preliminary set of resonance parameters and the related covariance matrix were derived in the energy range 


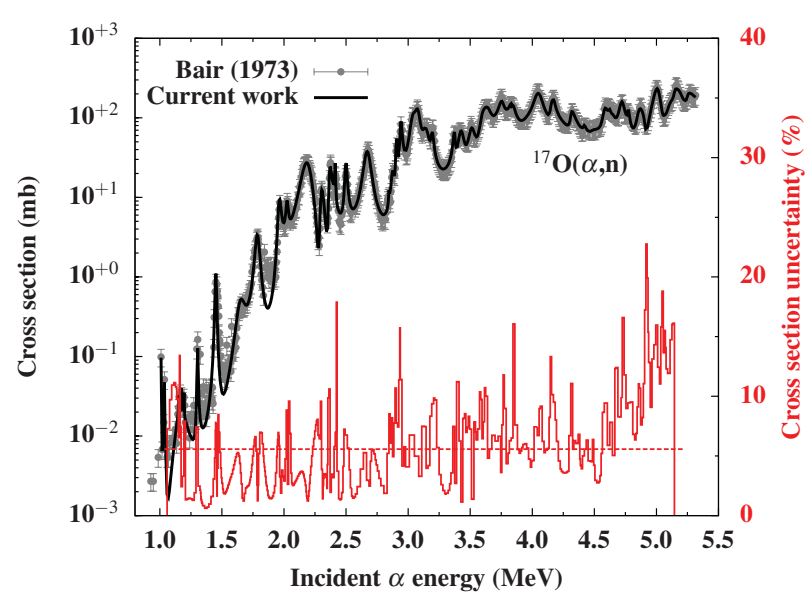

Figure 3. ${ }^{17} \mathrm{O}(\alpha, \mathrm{n})$ cross sections reconstructed from the preliminary set of resonance parameters in the energy range of 1-5 MeV compared with Bair's experimental data [9]. Relative cross section uncertainties (in percent) are shown in a continuous red line, along with their average relative uncertainty $(5.6 \%)$ in a dashed red line.

Table 1. ${ }^{17,18} \mathrm{O}(\alpha, \mathrm{n})$ experimental cross section data sets used in SAMMY calculations.

\begin{tabular}{|c|c|c|c|c|}
\hline Nuc./Ref. & $\mathrm{E}_{\alpha}(\mathrm{MeV})$ & Unc. $(\%)$ & $\mathrm{N}^{\mathrm{a}}$ & Unc. $(\%)^{\mathrm{b}}$ \\
\hline${ }^{17} \mathrm{O}[9]$ & $0.92-5.31$ & $25^{\mathrm{c}}$ & 1.35 & 7 \\
\hline${ }^{18} \mathrm{O}[8]$ & $1.06-2.50$ & $25^{\mathrm{d}}$ & 1.35 & 7 \\
\hline${ }^{18} \mathrm{O}[9]$ & $2.43-5.14$ & 25 & 1.35 & 7 \\
\hline${ }^{\text {nat }} \mathrm{O}[10]$ & $4.62-4.8^{\mathrm{e}}$ & 7 & 1.00 & 7 \\
\hline \multicolumn{5}{|c|}{$\begin{array}{l}\text { a Normalization recommended by Bair to correct bias in thin-target } \\
\text { measurements. }\end{array}$} \\
\hline \multirow{2}{*}{\multicolumn{5}{|c|}{$\begin{array}{l}{ }^{b} \text { Uncertainty assigned to each data point after normalization as stated in [10]. } \\
\text { No correlation between data points was assumed. }\end{array}$}} \\
\hline \multicolumn{2}{|c|}{${ }^{\mathrm{c}}$ Excluding uncertainty of graphite sphere detector efficiency. } & & & \\
\hline \multicolumn{5}{|c|}{ d Assumed uncertainty since no error analysis was reported. } \\
\hline
\end{tabular}

between $0-5 \mathrm{MeV}$. The values of resonance parameters were derived from the fit of Bair's data [8,9]. As displayed in Table 1, the analysis of the ${ }^{18} \mathrm{O}$ isotope was based on two thin-target measurements of $(\alpha, \mathrm{n})$ cross section data. The first set covers the incident $\alpha$-energy range $1.06 \leq E_{\alpha} \leq 2.50 \mathrm{MeV}$, and the second covers $2.43 \leq$ $E_{\alpha} \leq 5.14 \mathrm{MeV}$. Bair's measurement for the ${ }^{17} \mathrm{O}$ isotope extends over the energy range $0.92 \leq E_{\alpha} \leq 5.31 \mathrm{MeV}$. The reported overall uncertainty for ${ }^{17}, 18 \mathrm{O}$ experimental data sets [9] measured up to about $5 \mathrm{MeV}$ was reported as at least $25 \%$. The same percentage of uncertainty was assumed for the ${ }^{18} \mathrm{O}$ data set [8] measured at $\alpha$ energies between 1 and $2.5 \mathrm{MeV}$, since no error analysis was reported. Based on Bair's recommendation, the lowerenergy ${ }^{17,18} \mathrm{O}(\alpha, \mathrm{n})$ cross sections were scaled by a large normalization factor of 1.35 applied to all thin-target data sets. This normalization of cross sections was performed on the basis of Bair's thick-target measurements of ${ }^{\text {nat }} \mathrm{O}(\alpha, \mathrm{n})[10]$ that had much smaller uncertainty. After these data sets were normalized, a new error of about $7 \%$ was assigned as stated in [10]. Figures 3 and 4 show the ${ }^{17,18} \mathrm{O}(\alpha, \mathrm{n})$ cross section reconstructed from the preliminary set of resonance parameters in good agreement with the experimental data sets of Table 1. In the analyzed energy range, as the result of the Bayesian update procedure used to update the preliminary set of parameters by taking into account the experimental data

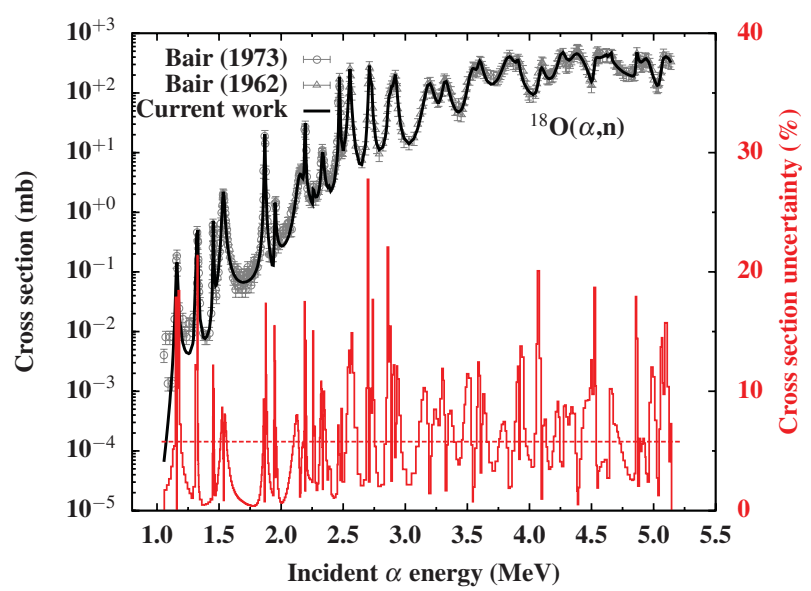

Figure 4. ${ }^{18} \mathrm{O}(\alpha, \mathrm{n})$ cross sections reconstructed from the preliminary set of resonance parameters in the energy range of 1-5 MeV compared with Bair's experimental data $[8,9]$. Relative cross section uncertainties (in percent) are shown in a continuous red line, along with their average relative uncertainty $(5.8 \%)$ in a dashed red line.

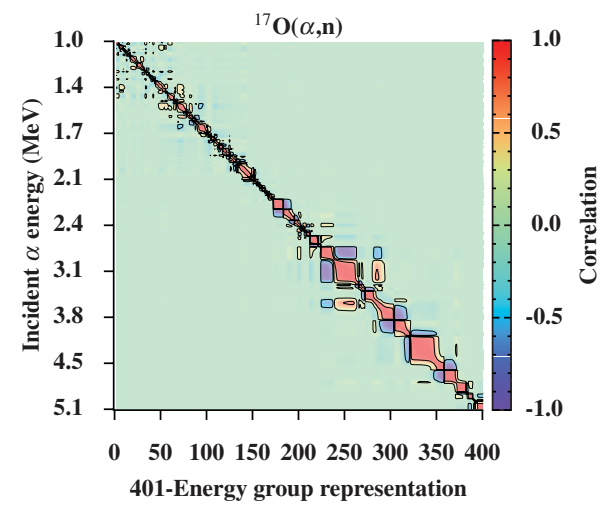

Figure 5. Correlation matrix of ${ }^{17} \mathrm{O}(\alpha, n)$ cross sections in 401energy group representation. Correlations below $1 \%$ are not displayed.

sets, the relative cross section uncertainties are shown (in a red continuous line) on average $5.6 \%$ and $5.8 \%$ for ${ }^{17} \mathrm{O}$ and ${ }^{18} \mathrm{O}$, respectively (dashed red line).

It should be noted that, in the experimental data sets analyzed, up to $5 \mathrm{MeV}$ the partial components of the $(\mathrm{n}, \alpha)$ cross sections related to the several excited states were convoluted. With more and better informative data [11], the values of the resonance widths related to each excited states could be more accurate and the fit improved. Moreover, additional data are also needed to accurately determine the partial widths for the ${ }^{17} \mathrm{O}(\alpha, \alpha)$ channel. For ${ }^{18} \mathrm{O}$ we relied on the work of Goldberg et al. [12].

Figures 5 and 6 also show the cross section correlation matrices of ${ }^{17,18} \mathrm{O}$ in 401 -energy group representation. Long-range correlations seem to be negligible and only a few neighbor resonances have strong negative or positive correlation.

\section{Application}

The covariance information related to ${ }^{17,18} \mathrm{O}(\alpha, \mathrm{n})$ cross sections was propagated to neutron source generation in an investigation using the ORIGEN code for the case of a 


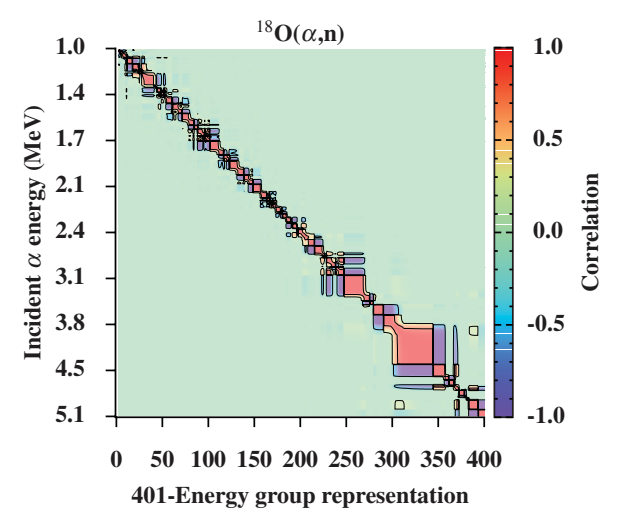

Figure 6. Correlation matrix of ${ }^{18} \mathrm{O}(\alpha, n)$ cross sections in 401energy group representation. Correlations below $1 \%$ are not displayed.

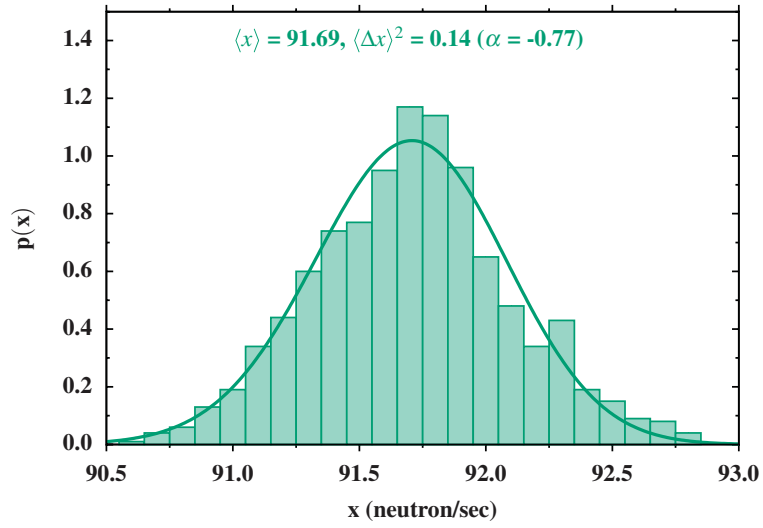

Figure 7. Normalized distribution related to the number of neutron per second obtained by ORIGEN calculations. The fit of a skew normal distribution (solid line) is also shown.

typical plutonium oxide matrix $\left(\mathrm{PuO}_{2}\right)$ composed by $1 \mathrm{~g}$ of plutonium (82.8\% of total isotopic composition).

The normalized distribution related to the number of neutrons per second shown in Fig. 7 was generated by performing 1,000 ORIGEN calculations, each time randomly sampling cross section libraries generated on the basis of the cross section and covariance information described in the Sect. 3 and in Ref. [6]. The set of libraries was generated by random perturbation factors obtained from the multivariate (normal) distribution based on the covariance information relative to the evaluated
${ }^{17,18} \mathrm{O}(\alpha, \mathrm{n})$ cross sections. An attempt was made to fit the obtained distribution with a skew normal distribution (solid line). The location parameter $\mu=\langle x\rangle$, along with its variance and the shape parameter $\alpha$, are reported. The total uncertainty in the computed neutron source due to $(\alpha, \mathrm{n})$ is about $0.4 \%$.

\section{Conclusion}

The $R$-matrix SAMMY implementation was applied using the Reich-Moore approximation to determine a consistent, albeit preliminary, set of resonance parameters and related covariance information for $\alpha+{ }^{17,18} \mathrm{O}$ in the energy range up to $5 \mathrm{MeV}$.

In the future, these evaluations of resonance parameters can be finalized and the analysis of their systematics improved by including a more comprehensive set of experimental data sets.

This work was motivated by the need to assess uncertainty in the neutron generation rates due to uncertainty of basic nuclear data. Following the work in Ref. [6], in which a self-consistent and rigorous methodology were developed to propagate nuclear covariance data to neutron source calculations for a typical uranium oxide fuel, in this paper the same methodology was applied to a $\mathrm{PuO}_{2}$ matrix.

This work was supported by the U.S. Department of Energy, National Nuclear Security Administration Office of Defense Nuclear Nonproliferation Research and Development.

\section{References}

[1] M.B. Chadwick et al., Nuclear Data Sheets 112, 2887 (2011)

[2] I.C. Gauld et al., Nucl. Tech. 174, 169 (2011)

[3] W.B. Wilson et al., Report LA-UR-02-1839 (2002)

[4] R.T. Perry et al., Report LA-8869-MS (1981)

[5] N.M. Larson, Report ORNL/TM-9179/R8 (2008)

[6] M.T. Pigni et al., Prog. Nucl. Energy 91, 147 (2016)

[7] R. Babut et al., Nucl. Sci. Eng. 151, 135 (2005)

[8] J.K. Bair et al., Phys. Rev. 128, 299 (1962)

[9] J.K. Bair et al., Phys. Rev. C7, 1356 (1973)

[10] J.K. Bair et al., Nucl. Sci. Eng. 71, 18 (1979)

[11] A. Best et al., Phys. Rev. C87, 045806 (2013)

[12] V.Z. Goldberg et al., Phys. Rev. C69, 024602 (2004) 